\title{
Inventario florístico de un bosque pluvial tropical (bp-T) en Manungará, municipio de Tadó, Chocó, Colombia
}

\section{Floristic inventory of a rain tropical forest (bp-T) in Manungará, Tadó municipality, it Chocó, Colombia}

\section{Yarleidys Cruz Mosquera*}

\section{Resumen}

Se presentan los resultados del inventarioflorístico realizado en el año 2007 en el corregimiento de Manungará, municipio de Tadó, Se establecieron dos subparcelas de 0,1 h cada una; la subparcela $A$ correspondió a una bosque sin intervención minera y la subparcela $B$ a un bosque intervenido por minería, donde se censaron todos los individuos con $D A P \geq 2,5 \mathrm{~cm}$, obteniendo como resultado 354 individuos representados en 117 especies, 96 géneros y 32 familias de las cuales cinco son indeterminadas; en la subparcela A se registraron 277 individuos, agrupados en 71 especies, 70 géneros y 29 familias y en la subparcela B se registraron 77 individuos, 46 especies, 41 géneros y 24 familias. Las familias más abundantes en relación con el número de individuos en ambas subparcelas fueron: Arecaceae 42, Melastomataceae 38, Euphorbiaceae 34, Moraceae 24, Rubiaceae 21 y Myristicaceae 20 individuos respectivamente. Las familias con menos individuos fueron: Ochnaceae 9, Lecytidaceae 8, Olacaceae 3 y Meliaceae con 2 individuos respectivamente. El área basal investigada fue de 15.6468 $\mathrm{m}^{2} / 0.1 \mathrm{ha}$, siendo el mayor aporte diamétrico de $70 \mathrm{~cm}$ para las especies Alchorneapsis floribunda (Benth) de la familia Euphorbiaceae y la especie Couma macrocarpa Bord.rod de la familia Apocynaceae.

Palabras clave: Manungará, Índice, Individuos.
* Bióloga con énfasis en recursos naturales, Contratista Instituto de Investigciones Ambientales del Pacífico(IIAP), Quibdó, Colombia. e-mail: yacrumo82@gmail.com

Recibido: 3 de diciembre de 2013 Aprobado: 1 de marzo de 2014

\begin{abstract}
Results from floristic inventory carried out from year of 2007 in Manungará s, Tado' 's municipality, two established sub plots of 0,1 h each one the subplot to corresponds to one forest without mining intervention and the sub parcels $B$ to a forest controlled by mining industry, where all the individuals were registered by $D A P>2,5 \mathrm{~cm}$, obtaining as result 354 individuals represented in 117 species, 96 kinds and 32 families of which five are indeterminate; in the sub it parcels to there registered 277 individuals grouped in 71 species, 70 kinds and 29 families and in the sub plot B there registered 77 individuals, 46 species, 41 kinds and 24 families. The most abundant families with relation to the number of individuals in both subplots are Arecaceae.
\end{abstract}

Keywords: Manungará, Index, Individuals.

\section{Introducción}

El Chocó Biogeográfico se considera como uno de los ecosistemas más complejos del país y de mayor importancia económica no solo por su riqueza de especies sino también por el aporte maderable que hace a la economía colombiana (Del Valle 1993). Es por ello que en este territorio predomina el bosque pluvial tropical, que se caracteriza por los niveles de precipitación anual extremadamente elevados (cerca de 1200 m); debido a esto el departamento del Chocó es una de 


\section{Bioetnia Volumen 11, 2014}

las zona más húmedas del mundo y al mismo tiempo, una de las más ricas en especies de flora y fauna por lo que se calcula entre siete mil a ocho mil especies de plantas de las 45.000 que existen en Colombia (Forero y Gentry 1989); varias de estas especies, inclusive géneros completos, son endémicos del departamento del Chocó, siendo este un importante centro de origen y distribución de flora hacia toda la región Pacífica (Gentry 1982).

La región del San Juan y sus afluentes está cubierta de selva super humedad encerrada entre la cordillera occidental, la serranía del Baudó, el Istmo de San Pablo, el macizo de los farallones de Cali, siendo menos pantanosa que el Atrato porque sus ríos, son en su mayoría, encajonados. En las orillas de las ollas media y baja del río, se encuentran pequeños cultivos de maíz, plátano, caña de azúcar, pero a la vez realizan actividades de explotación maderable mientras que en la parte alta de esta zona es fundamentalmente minera (Mosquera 1992). En la actualidad en la región del San Juan se viene desarrollando la extracción de metales preciosos con ayuda de retroexcavadoras, dragas y bombas de inyección, tecnologías que produce la pérdida de especies vegetales y destrucción de grandes cantidades de microhábitat de muchos vertebrados (Leal 1995), al mismo tiempo ha producido fenómenos de violencia, pérdida de identidad cultural y pérdida de empoderamiento de los grupos étnicos en sus territorios (Ayala 2005). Por lo anterior el Instituto de Investigaciones Ambientales del Pacífico (IIAP) ha venido consolidando su misión investigativa en el departamento del Chocó. Bajo esta dirección el IIAP viene trabajando en la implementación de la Estación Ambiental del Alto San Juan (EAASJ), lugar donde se desarrollan investigaciones en diferentes niveles con participación comunitaria en aras de conservar la biodiversidad del Chocó Biogeográfico.

Por consiguiente se realizó el inventario florístico de un bosque pluvial tropical en el corregimiento de Manungará, municipio de Tadó, Chocó, como herramienta básica para comprobar el estado actual y diversidad florística, con miras a desarrollar estrategias que permitan contribuir al conocimiento de los recursos vegetales con utilidad para las comunidades afrodescendientes asentadas en la zona de estudio.

\section{Metodología}

Área de estudio. El corregimiento de Manungará, municipio de Tadó, se encuentra ubicado en la región del Alto San Juan, en la zona de vida selva pluvial tropical (bp-T). Se localiza a los $5^{\circ} 16^{\prime}$ de latitud norte y $76^{\circ} 13^{\prime}$ al oeste de Greenwich, limitando al norte con la vereda de Bogare, al sur con el balneario de Pueblo Viejo, al oriente con el corregimiento de Tapón y al occidente con la vereda de Chacuato (Figura 1). Posee una precipitación anual entre 1600 y $6800 \mathrm{~mm}$ al año, la temperatura oscila entre $27^{\circ} \mathrm{C}$ y $29^{\circ} \mathrm{C}$, con una humedad relativa entre $70 \%$ a $85 \%$ (Rodríguez e Ibargüen 2004).

Se seleccionaron dos áreas con diferentes grados de intervención antrópica, las cuales se denominaron subparcelas A un bosque secundario denso y subparcela B un área de explotación minera en recuperación (Figura 2).

Métodos. Para la ejecución del presente estudio se implementó la metodología propuesta por Gentry (1986) y mejorada por Galeano (2000), en donde se realizaron 10 transeptos de ( 2 x $50 \mathrm{~m})$, tanto en la subparcela A (Figura 1) como en la subparcela B (Figura 2); se ejecutaron las respectivas colectas de todos los árboles y arbustos, con diámetros a la altura del pecho (DAP) de $\geq 2.5 \mathrm{~cm}$, tomando tres ejemplares por cada individuo, anotando sus respectivos datos morfológicos como: látex, olor, exudado y otras características que permitieran facilitar su identificación en cuanto a familia, género y especie.

Identificación taxonómica de las colecciones. La determinación del material colectado se identificó por medio de confrontación con el material del Herbario CHOCÓ de la Universidad Tecnológica del Chocó. La información se complementó con el uso de literatura especializada (Gentry 1993, Mahecha 1997).

Análisis de los datos. Para la determinación de la estructura de las áreas estudiadas se cuantifican las siguientes variables: abundancia absoluta y relativa, frecuencia absoluta y relativa, dominancia absoluta y relativa e índice de valor de importancia (IVI). Las clases diamétricas de determinaron teniendo en cuenta el diámetro de individuos arbóreos utilizando intervalos de $10 \mathrm{~cm}$, además se consideraron las siguientes variables:

$$
\begin{gathered}
\text { Índice de Shannon Weaver: } \\
\mathrm{H}^{\prime}=(\mathrm{N} \log \mathrm{N})-(\mathrm{ni} \log \mathrm{ni}) / \mathrm{N} \\
\text { Índice de Simpson: } \\
\mathrm{Ds}=1-\sum(\mathrm{ni})(\mathrm{ni}-1) / \mathrm{N}(\mathrm{N}-1) \\
\text { Índice de Margalef: } \\
\text { Dmg }=(\mathrm{s}-1) / \operatorname{logn} \mathrm{N}
\end{gathered}
$$

\section{Resultados y discusión}

Composición florística. Se registró un total de 354 individuos con DAP $\geq 2.5 \mathrm{~cm}$ representados en 117 especies (cinco fueron indeterminadas), 96 géneros y 38 familias, de las cuales en la subparcela A se obtuvieron 265 individuos agrupados en 32 familias, 70 géneros y 71 especies y en la B se obtuvieron 89 individuos agrupados en 29 familias, 41 géneros y 46 especies, donde se manifestaron hábitos de crecimiento en árboles, arbustos y palmas. Las familias mejor representadas en relación con el número de indivi- 


\section{Inventario florístico de un bosque pluvial tropical. Y Cruz}

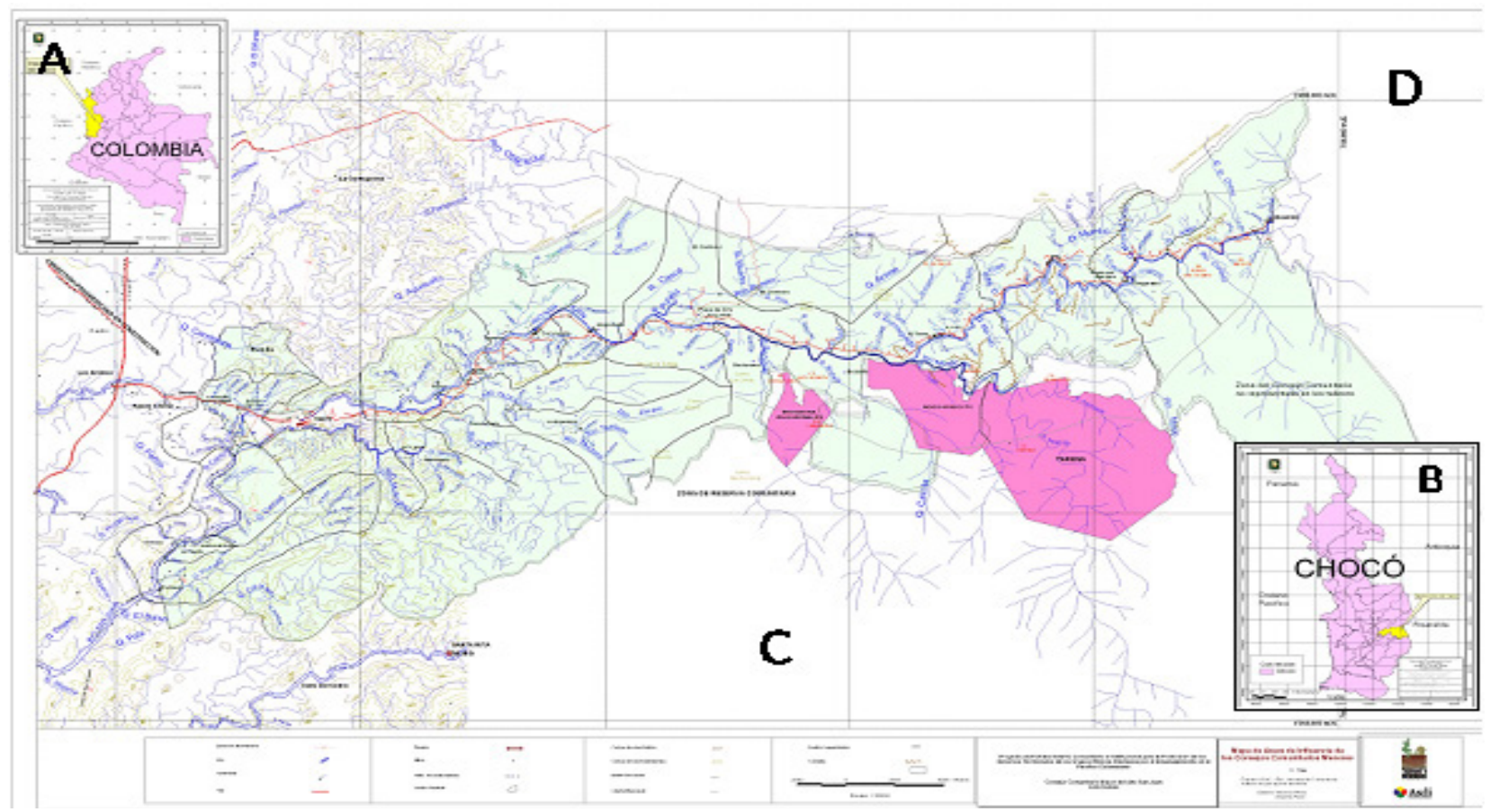

Figura 1. Municipio de Tadó. (A) República de Colombia. (B) Departamento del Chocó. (C) Municipio de Tadó, Territorio colectivo de la Asociación Campesina del Alto San Juan (ASOCASAN).

(D) Corregimiento de Manungará.
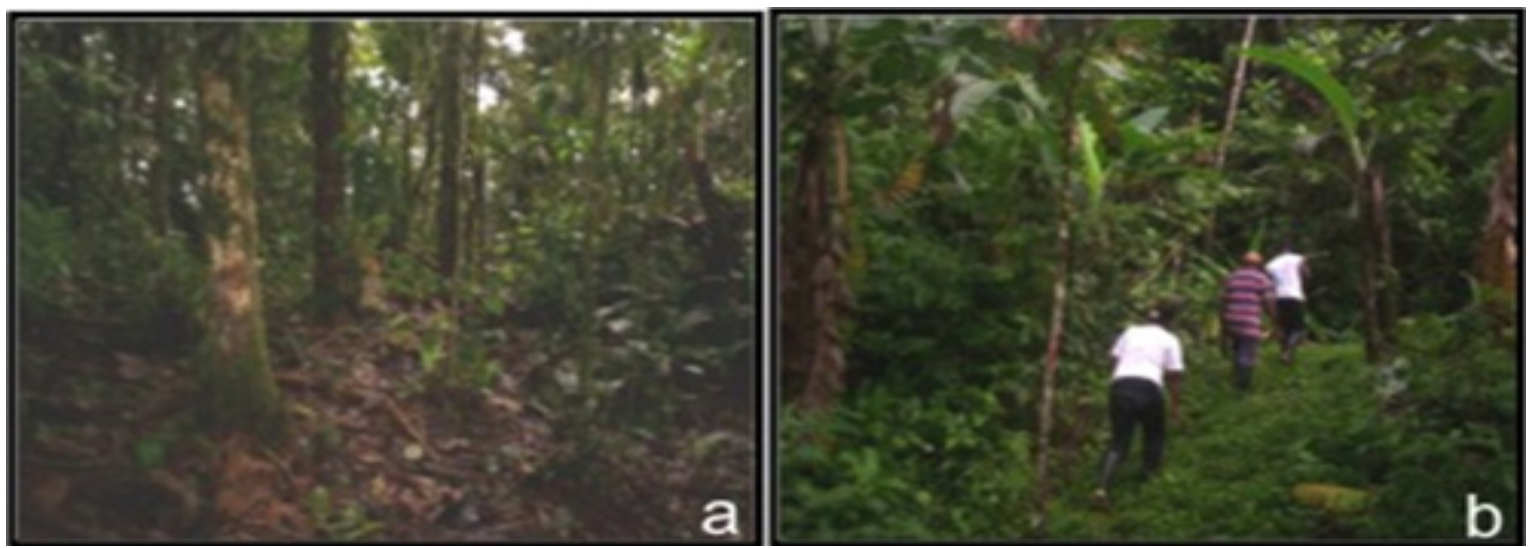

Figura 2. Ubicación de las zonas de monitoreo en el corregimiento de Manungará. a: Bosque segundario denso, b: Área de explotación minera en recuperación.

duos en las parcelas fueron Arecaceae con 42 (11.8\%), Melastomataceae con $38(10.7 \%)$ y Euphorbiaceae con $34(9.6 \%)$ (Figura 3).

Sin lugar a duda, la composición florística de este bosque es típica de bosque húmedo poco intervenido donde predominan familias botánicas como Rubiaceae, Arecaceae, Moraceae, Melastomataceae, Annonaceae, Clusiaceae, entre otras, las cuales caracterizan este tipo de bosque. Los resultados de este estudio coinciden parcialmente con lo reportado para los bosques del Chocó por Ramos y Palacios
(1999), Palacios (2002), Cárdenas (2003), Galeano (2002), García et al. 2002, 2004), Mena (2003), Rodríguez e Ibargüen (2004). Coincidiendo en gran manera con las familias botánicas encontradas se destacan: Moraceae, Arecaceae, Melastomataceae, Rubiaceae, Euphorbiaceae y Lauraceae. A pesar de que son zonas diferentes, así como también los diámetros y hectáreas utilizadas, sus bosques tienen una gran similitud en cuanto a la cobertura boscosa debido a que se encuentran en proceso de recuperación después de intervenciones humanas (causantes de tala de árboles, 


\section{Bioetnia Volumen 11, 2014}

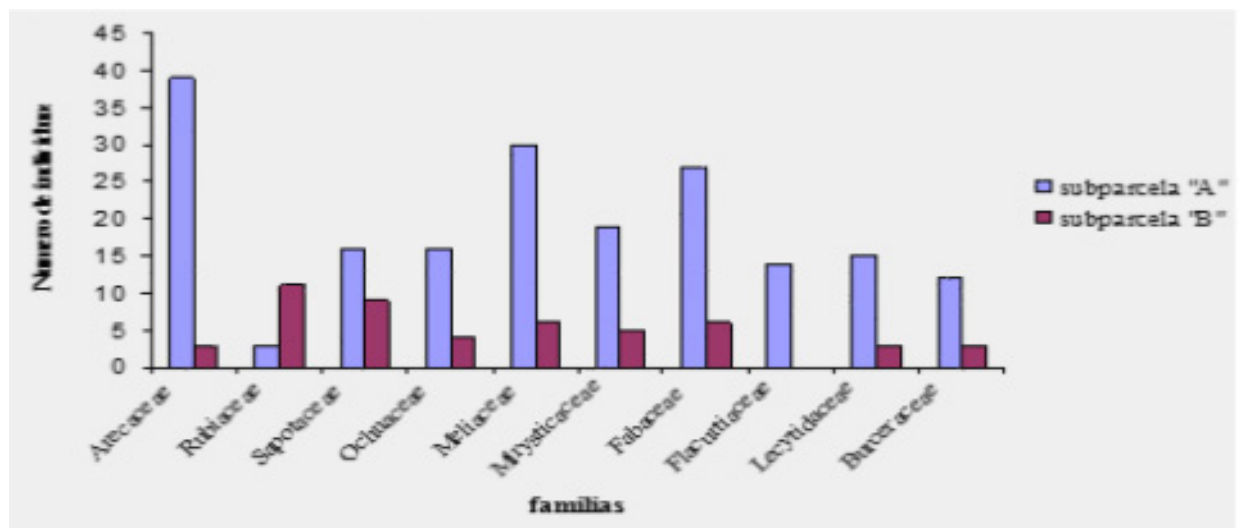

Figura 3. Familias más representativas en las parcelas $A$ y $B$ en el corregimiento de Manungará, Tadó.

derrumbes, etc.); las familias botánicas que priman en ellos son especies leñosas, las cuales se pueden adaptar mejor a las condiciones que el bosque les brinda (Melgar 2001).

Un aspecto que vale la pena resaltar es la notable presencia de palmas en estos bosques, lo que estaría coincidiendo con lo señalado por Gentry (1988), cuando afirma que una alta representación de palmas es típica de bosques lluviosos en el neotrópico, las cuales en sitios abiertos compensan la escasez de especies con un número grande de individuos. A diferencia de Bernal y Galeano (1993), opinan que la presencia de palmas en los ecosistemas del Pacífico, influye en la maraña de interacciones, aun no bien comprendidas que ocurre entre las palmas y la fauna. Las palmas proveen de alimento y albergan a una gran cantidad de animales, mucho de los cuales, actúan a su vez como polinizadores y dispersores de semillas.

Medidas de diversidad. La riqueza de especies arrojó valores de 12,45 para la subparcela A (bosque segundario denso) y 10,36 para la subparcela B (área de explotación minera en recuperación) respectivamente. La diversidad por su parte, fue de 3.96 subparcela A y 3.70 subparcela $\mathrm{B}$ respectivamente, valores que indudablemente indican una alta diversidad coincidiendo así con los resultados alcanzados por Palacios (2002); igualmente se observó que el bosque secundario denso A es más rico y diverso que el área de explotación minera en recuperación $\mathrm{B}$ porque posee un mayor número de especies e individuos, resultados que a la vez permiten resaltar lo expuesto por Berry (2002) cuando manifiesta que la riqueza de especies en los bosques tropicales no dependen únicamente del área muestreada, sino también del número de individuos que se encuentran en esa área.

Al comparar la diversidad presentada para las dos zonas estudiadas con resultados obtenidos por otros autores en diferentes lugares del Chocó (Tabla 1) se puede apreciar que no supera valores alcanzados en los trabajos realizados por Galeano (2002), Palacios (2002), Gentry (1986) y Franco et al. (1997); sin embargo los resultados de esta investigación se encuentran dentro del promedio establecido por Gentry (1992) para los bosques del neotrópico.

Estructura diamétrica. Los 354 individuos del corregimiento de Manungará presentaron un área basal de 15.6468 $\mathrm{m}^{2} / 0.1$ ha, siendo el mayor aporte diamétrico de $70 \mathrm{~cm}$ para las especies Alchorneapsis floribunda (Benth) de la familia Euphorbiaceae y la especie Couma macrocarpa Bord.rod de la familia Apocynaceae.

En relación con el promedio diamétrico se puede afirmar que los bosques son regulares, aspecto que corrobora lo expuesto por Gentry (1993) cuando señala que los bosques del Chocó presentan una estructura única en muchos sentidos, caracterizándose por alta densidad de árboles pequeños y medianos (2.5-30 cm de DAP), aspecto que según Melgar (2001), se debe a la caída de uno o varios árboles provocada por la acción de fuertes vientos, deslizamiento del terreno o por la dinámica vegetal natural del bosque, al igual que por la alta precipitación.

La distribución de los individuos por clases diamétricas mostró un comportamiento similar a una curva en forma de "J "invertida para las dos áreas de monitoreo, donde el $31 \%$ y el $21 \%$ de los individuos, estuvieron en la clase I (1-10 cm DAP) (Figura 4).

Los bosques están conformados por gran cantidad de individuos jóvenes con pocos árboles maduros, producto de una lenta recuperación luego de haber soportado la extracción selectiva de madera, característica que hace que la distribución diamétrica tenga esta forma. Esto es corroborado por Lamprecht (1990) cuando afirma que la distribución diamétrica en bosques nativos jóvenes o en proceso de recuperación presenta una tendencia en forma de "J" invertida. Pardo et al. (1994), consideran que la distribución diamétrica en forma de "J" invertida de los individuos en los bosques del Chocó puede ser el resultado de la interacción de factores como suelos permanentemente lavados y con bajo contenido de nutrientes que no permite 


\section{Inventario florístico de un bosque pluvial tropical. Y Cruz}

Tabla 1

Comparación de la diversidad florística de los bosques del corregimiento de Manungará, Tadó, con otros de la región pacífica colombiana

\begin{tabular}{|c|c|c|c|c|c|c|c|c|}
\hline \multirow[t]{2}{*}{ Autor/año } & \multirow[t]{2}{*}{ Sitio } & \multicolumn{7}{|c|}{ Número de } \\
\hline & & \multicolumn{3}{|c|}{ individuos especies familias } & \multicolumn{4}{|c|}{ Shannon Margalet Área (ha) DAP (cm } \\
\hline \multirow[t]{2}{*}{ Galeano (2002) } & Coquí & 471 & 158 & 38 & - & - & 0,4 & $\geq 5$ \\
\hline & Nuquí & 414 & 151 & 42 & - & - & 0,4 & $\geq 5$ \\
\hline \multirow[t]{2}{*}{ Palacios (2002) } & Lloró & 886 & 83 & 33 & 3,59 & 12,08 & 0,15 & $\geq$ \\
\hline & Pacurita & 1003 & 105 & 32 & 3,87 & 15,04 & 0,15 & $\geq$ \\
\hline Martínez y Alvarado (2000) & Serranía del Baudó & - & 133 & 40 & - & - & 0,1 & $\geq 2,5$ \\
\hline \multirow[t]{2}{*}{ Gentry (1986) } & Tutunendo (Chocó) & 527 & 258 & 57 & - & - & 0,1 & $>2,5$ \\
\hline & $\begin{array}{l}\text { Bajo Calima (Valle } \\
\text { del Cauca) }\end{array}$ & 497 & 265 & 60 & - & - & 0,1 & $>2,5$ \\
\hline \multirow[t]{2}{*}{ Franco et al. (1997) } & Campurcaná & 1444 & 269 & 69 & 0,82 & - & 0,1 & $>2,5$ \\
\hline & Ñambi & 1347 & 205 & 59 & 0,87 & - & 0,1 & $>2,5$ \\
\hline \multirow[t]{2}{*}{ Becerra y Cruz 2007} & $\begin{array}{l}\text { Bosque segundario } \\
\text { denso subparcela A }\end{array}$ & 265 & 71 & 32 & 3,96 & 12,45 & 0,1 & $>2,5$ \\
\hline & $\begin{array}{l}\text { Área de explotación } \\
\text { minera en recuperación } \\
\text { subparcela B }\end{array}$ & 89 & 46 & 29 & 3,70 & 10,36 & 0,1 & $>2,5$ \\
\hline
\end{tabular}

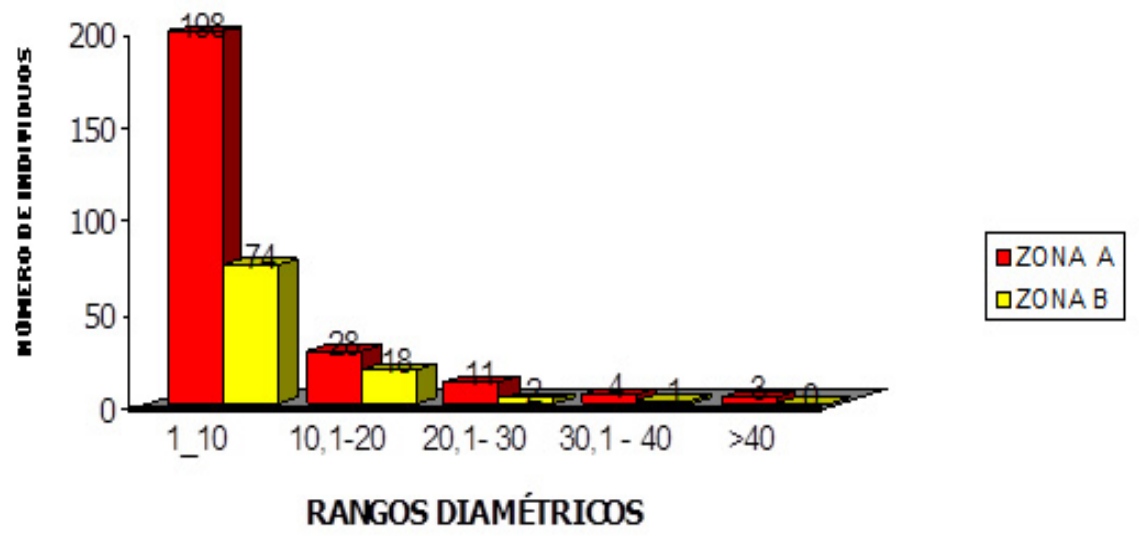

Figura 4. Distribución diamétrica de los individuos de los bosques de un bosque segundario denso A y un área de explotación minera en recuperación $\mathrm{B}$.

el sostenimiento de árboles de tamaño grande, la topografía escarpada del terreno que influye sobre la dinámica del bosque y favorece la presencia de individuos con diámetros menores.

Importancia ecológica de las especies (IVI). De acuerdo con las áreas inventariadas se logra observar que las diez primeras especies que representan $78.7 \%$ del total del IVI (305) y la dominancia de las mismas equivale a $45.5 \%$ del área basal del bosque $\left(15.648 \mathrm{~m}^{2} / 0.1 \mathrm{ha}\right)$, mientras que las
344 restantes representan $26.8 \%$ del IVI y $60.6 \%$ del área basal total $\left(15.648 \mathrm{~m}^{2} / 0.1 \mathrm{ha}\right)$. El hecho de que sean pocas especies que representan la mayoría del porcentaje del valor de importancia (Tabla 2) indica que este pequeño grupo está dominando la mayor parte del espacio y de los recursos presentes en el subsuelo de la zona. Galeano (2002) manifiesta que la dominancia de unas pocas especies puede estar ligada a una baja riqueza y una alta disponibilidad de nutrientes. A pesar que la mayor parte de las especies 


\section{Bioetnia Volumen 11, 2014}

Tabla 2

Índice de valor de importancia (IVI) para las diez especies $2.5>\mathrm{cm}$ DAP más importantes de un bosque secundario denso y un área de explotación minera en recuperación en el corregimiento de Manungará, Tadó, Chocó, Colombia

\begin{tabular}{lrrrrrrr}
\hline Bosque secundario denso & Aa & Ar\% & Fa & Fr\% & Da & Dr\% & IVI \\
\hline Especies & & & & & & & \\
\hline Pouroma chocuana Standl & 4 & 1,5 & 40 & 1,5 & 1,218 & 14,1 & 17,1 \\
\hline Alchorneapsis floribunda (Benth) & 2 & 0,8 & 20 & 0,8 & 1,316 & 15,2 & 16,7 \\
Licania cf macrocarpa Cuart & 2 & 0,8 & 20 & 0,8 & 1,109 & 12,8 & 14,3 \\
\hline Mabea ocidentalis Benth & 9 & 3,4 & 90 & 3,4 & 0,407 & 4,7 & 11,5 \\
\hline Brosimum utile (HBK) Pittier & 15 & 1,5 & 150 & 1,5 & 0,543 & 6,2 & 9,2 \\
\hline Virola elongate Benth Warb & 4 & 1,1 & 40 & 1,1 & 0,551 & 6,4 & 8,6 \\
Ossaea sp & 8 & 2,3 & 80 & 2,3 & 0,353 & 4,1 & 8,6 \\
\hline Leonia trianda Cuart & 7 & 2,6 & 70 & 2,6 & 0,161 & 1,9 & 7,1 \\
Otoba latialata pitt & 4 & 1,1 & 40 & 1,1 & 0,417 & 4,8 & 7,1 \\
\hline Miconia nervosa Sm & 7 & 2,6 & 70 & 2,6 & 0,027 & 0,3 & 5,6 \\
Subtotal (10 especies) & 62 & 17,7 & 620 & 17,7 & 6,102 & 70,4 & 105,8 \\
\hline Restantes 61 especies & 203 & 72.6 & 2030 & 72,6 & 3,668 & 42,6 & 187,9 \\
Total 71 especies & 265 & 100,0 & 2650 & 100,0 & 9,77 & 100,0 & 300,0 \\
\hline
\end{tabular}

\section{Área de explotación minera}

en recuperación

\begin{tabular}{lrrrrrrrr|r} 
Couma macrocarpa Bord & 2 & 2,2 & 20 & 2,2 & 1,263 & 20,1 & 24,5 \\
Apeaba aspera abull & 3 & 3,3 & 30 & 3,3 & 0,794 & 14 & 20,6 \\
Miconia nervosa Sm & 2 & 4,4 & 20 & 4,4 & 0,139 & 2 & 10,8 \\
Schefflera sp & 1 & 1,1 & 10 & 1,1 & 0,394 & 7,8 & 10 \\
Mabea occidentales Benth & 3 & 3,3 & 30 & 3,3 & 0,072 & 1,4 & 8 \\
\hline Ladenbergia muzonensis & 3 & 3,3 & 30 & 3,3 & 0,049 & 0,92 & 7,5 \\
Eschweilera Nov & 2 & 2,2 & 20 & 2,2 & 0,153 & 3 & 7,4 \\
\hline Humiriaceae NNI & 3 & 3,3 & 30 & 3,3 & 0,037 & 0,71 & 7,3 \\
Piptademia aff speciosa & 3 & 3,3 & 30 & 3,3 & 0,032 & 0,65 & 7,2 \\
\hline Tocota spadiciflora Triana & 2 & 2,2 & 20 & 2,2 & 0,142 & 2,75 & 7,1 \\
\hline Subtotal (10 especies) & 22 & 26,4 & 220 & 26,4 & 2,933 & 50,58 & 110,6 \\
Restantes 36 especies & 67 & 80,3 & 670 & 80,3 & 2,017 & 36,17 & 189,4 \\
Total 46 especies & 89 & 100,00 & 890 & 100,00 & 4,95 & 100,00 & 300,00 \\
\hline
\end{tabular}

presentaron IVI muy bajos, se puede afirmar que los bosques son heterogéneos debido a que ninguna especie tiene dominancia relevante sobre otra.

El hecho que Pourouma chocuana Standl y Couma macrocarp Bord, hallan presentado una leve dominancia sobre las demás especies se puede atribuir a que no son aprovechadas por los habitantes de la comunidades adyacentes a estos bosques, por esta misma razón son encontradas con mayores DAP que otras especies. Aspecto que es ratificado por Arroyo et al. (1994) cuando citan que la intervención antrópica ocasionó modificaciones estructurales en la formación vegetal apreciándose la reducción notable de especies de importancia económica y la dominancia relativa de especies cuya madera posee poco valor en el mercado. 


\section{Inventario florístico de un bosque pluvial tropical. Y Cruz}

\section{Conclusiones}

Los datos obtenidos ponen de manifiesto que el inventario florístico en el área estudiada muestra la existencia de un bosque pluvial tropical donde predominan las especies y géneros de familias como Araceae, Melastomataceae, Euphorbiaceaey Moraceae, presentando una gran diversidad y abundancia de especies vegetales a pesar de ser una zona donde se vienen realizando actividades mineras, lo cual ha causado deforestación de zonas boscosas y deterioro de ecosistemas pero aún es representativa.

A pesar de que las dos áreas se encuentran en localidades relativamente cercanas, la subparcela A presentó una leve diferencia en términos de diversidad y riqueza que la subparcela B y al mismo tiempo denotándose altos índices ecológicos en la subparcela A en relación con la sub parcela $B$.

Los análisis estructurales de este tipo de bosque permiten el conocimiento amplio y detallado de la organización, ubicación e interacción de los individuos. En cuanto a la distribución de las especies con DAP $\geq 2.5 \mathrm{~cm}$, en $0.1 \mathrm{ha}$, la mayor cantidad de individuos se ubican en la clase diamétrica de 1 a $10 \mathrm{~cm}$ y pocos estuvieron representados en las clases mayores de $70 \mathrm{~cm}$.

\section{Literatura citada}

Arroyo VJE, Palacios J, Mena A, Mosquera MM, Ramos Y, Mosquera A, et al. 1994. Estructura de un bosque pluvial tropical (bp-T) intervenido (explotación selectiva de madera). Memorias del I Congreso Nacional sobre Biodiversidad Biopacífico, Universidad del Valle, Instituto de Estudios del Pacífico, Cali, Colombia. pp. 115-19.

Ayala H. 2005. Diagnóstico situacional de la minería artesanal y en pequeña escala desarrollada por afrocolombiano en territorios colectivos de comunidades negras en el Chocó Biogeográfico. Quibdó: Centro Internacional de Investigaciones para el Desarrollo, Instituto de Investigaciones Ambientales del Pacífico (IIAP).

Becerra TA, Cruz MY. 2007. Composición florística y etnobotánica de un bosque pluvial Tropical (bp-T) en el corregimiento de Manungará, municipio de Tadó, Chocó, Colombia. Trabajo de grado presentado como requisito parcial para optar el título de Biólogo con Énfasis en Recursos Naturales. Quibdó: Facultad de Ciencias Básicas, Universidad Tecnológica del Chocó "Diego Luis Córdoba".

Bernal RJ, Galeano G. 1993. Las palmas del Andén Pacífico colombiano. En Leyva P(ed.). Colombia Pacifico. Tomo I. Bogotá: Proyecto Biopacífico. pp. 221-31.

Berry PE. 2002. Diversidad y endemismo en los bosques neotropicales de Bajura. En: Guariguata MR, Kattan GH (eds.). Ecología de bosques neotropicales. Cartago: Libro Universitario Regional; pp. 83-96.

Cárdenas D. 2003. Inventario florístico en el centro del Cuchillo, Tapón del Darién colombiano. Caldasia 25 (1):101-17.

Del Valle JI. 1993. Silvicultura y uso sostenido de los bosques. Referencia especial a los guadales, Nariño. En: Leyva P (ed.). Colombia Pacífico. Tomo II. Bogotá: Fondo FEN; pp. 693-713.

Franco R, Betancur P, Fernández J, Alonso JL. 1997. Diversidad florística en dos bosques subandinos del sur de Colombia. Caldasia 19 (1-2): 205-34.

Forero E, Gentry A. 1989. Lista anotada de plantas del departamento del Chocó, $N^{o}$ 10. Bogotá: Biblioteca José Jerónimo Triana; 142 pp.
Galeano G. 2000. Forest use at the Pacific coast of Chocó, Colombia. A quantitative approach. Economic Botany. 54 (3): 358-76.

Galeano G. 2002. Estructura, riqueza y composición de plantas leñosas en el Golfo de Tribugá, Chocó, Colombia. Caldasia 23 (11): 2-11.

García FJ, Palacios Y, Ramos A, Mena J, Arroyo E, González M. 2002. Composición estructura y etnobotánica de un bosque pluvial Tropical (bp-T) en Alero, Chocó. Revista Institucional Universidad Tecnológica del Chocó DLC. 17: 3-9.

García CF, Moreno MM, Robledo D, Mosquera LJ, Duque LP. 2004. Composición y diversidad florística de los bosques de la cuenca hídrica del río Cabí, Quibdó, Chocó Revista Institucional, Universidad Tecnológica del Chocó. 22: 13-23.

Gentry AH. 1982 Neotropical floristic diversity. Ann Missouri Bot Garden. 69: 557-93.

Gentry AH.1986. Species richness and floristic composition of Chocó region plant Caldasia. 15: 71-5.

Gentry AH. 1988. Changes in plant community diversity and floristic composition on environmental and geographical gradients. Ann Missouri Bot Garden. 75: 1-74.

Gentry AH. 1992. Tropical forest biodiversity: Distributional patterns and their conservational significance. Oikos. 63 (1): 19-28.

Gentry AH. 1993. Riqueza de especies y composición florística de la comunidad de plantas de la región del Chocó. Una actualización. Tomo I. En: Colombia Pacífico. Bogotá: Proyecto Biopacífico.

Gentry AH. 1986. Species richness and floristic composition of Chocó region plant. Caldasia. 15: 71-5.

Lamprecht H. 1990. Silvicultura en los trópicos. Los ecosistemas forestales en los bosques tropicales y sus especies arbóreas; posibilidades y métodos para un aprovechamiento sostenido. Göttingen: Deutsche Gesellschaft fur Technische Zusammenarbeit (GTZ) GmbH. Rossdorf, TZ-Verl.-Ges.; Instituto de Silvicultura de la Universidad de Göttingen; 335 pp.

Leal C. 1995. Vade retro excavadora. La sed de oro siembra desiertos en el Pacífico. Rev Ecol Pol Medio Amb Cult. 20: 4-9.

Martínez X, Alvarado I. 2000. Riqueza, composición y distribución de especies de acuerdo con las formas de crecimiento en un bosque de la vertiente occidental de la serranía del Baudó, Chocó, Colombia. Memoria del VIII Congreso Latinoamericano de Botánica y II Colombiano, Bogotá, Colombia; 467 pp.

Melgar MI. 2001. Potencial para el desarrollo y el manejo de bosques secundarios latifoliados dentro del programa Escuela de Planificación Orgánica Evolutiva (EPOE). Disponible en: http://www.gestiopolis.com/ desarrollo-y-manejo-de-bosques-secundarios-latifoliados/

Mena CS. 2003. Estructura y composición florística de un bosque de colina del corregimiento de Pacurita, Quibdó, Chocó, Colombia. Trabajo de grado. Programa de Biología con énfasis en Recursos Naturales, Facultad de Ciencias Básicas, Universidad Tecnológica del Chocó, Quibdó.

Mosquera AO. 1992. Geografía e historia del Chocó. Quibdó: Promotores Editorial Autores Chocoanos; pp. 81.

Palacios DL. 2002. Muestreo diagnóstico de la regeneración natural en dos bosques pluviales tropicales (bp-T) en Quibdó y Lloró, Chocó. Tesis de grado. Programa de Biología con énfasis en Recursos Naturales, Facultad de Ciencias Básicas, Universidad Tecnológica del Chocó, Quibdó.

Pardo P, Marcos E, Cediel C. 1994. Composición y diversidad florística de los bosques del Cabo Corriente, costa pacifica del Chocó. Memorias del I Congreso Nacional sobre Biodiversidad, Proyecto Biopacífico. -Universidad del Valle, Instituto de Estudos del Pacífico, Cali, Colombia. pp. 85-91.

Rodríguez CL, Ibargüen A. 2004. Caracterización estructural de la cobertura vegetal del bosque pluvial tropical en la estación ambiental del Instituto de Investigaciones Ambientales del Pacifico, corregimiento de Playa de Oro, municipio de Tadó, Chocó, Colombia. Tesis de grado. Programa de Biología con énfasis en Recursos Naturales, Facultad de Ciencias Básicas, Universidad Tecnológica del Chocó, Quibdó.

Ramos PYA, Palacios JC. 1999. Estructura de un bosque pluvial tropical (bpT) en Salero (Tadó, Chocó). Tesis de grado. Programa de Biología con énfasis en Recursos Naturales, Facultad de Ciencias Básicas, Universidad Tecnológica del Chocó, Quibdó. 